\title{
Efficient analysis of thick curved metal sheets
}

\author{
Eduard Ubeda ${ }^{1}$, Juan M. Rius ${ }^{1}$ \\ ${ }^{1}$ AntennaLAB, Department of Signal Theory and Communications, \\ Universitat Politecnica de Catalunya (BarcelonaTECH), Barcelona, Spain, ubeda@tsc.upc.edu
}

\begin{abstract}
The scattering analysis of perfectly conducting plates with the method-of-moment discretization of the Electric-Field Integral Equation has been traditionally carried out according to the thin-sheet approximation, which neglects the current contributions over the plate rims. The plate is then modelled as an open surface and handles a restricted number of unknowns as compared with the canonical modelling as a closed object. The thin-sheet approximation, although used in many practical applications, becomes inaccurate for thick enough plates or plates under plane wave incidence at grazing angles. Recently, a thick-plate approach has been presented to address the time-efficient and accurate scattering analysis of thick plates. This scheme exploits the symmetry properties of fields and currents thereby giving rise to two uncoupled problems with roughly half the number of unknowns of the original problem. In this paper, we extend this thick-plate scheme to the efficient scattering analysis of thick curved sheets.
\end{abstract}

Index Terms - Integral equations, method of moments.

\section{INTRODUCTION}

The scattering analysis of conducting (PEC) sheets with very small thickness is normally carried out through the thinsheet approximation [1], which arises from the application of the surface equivalence principle when the sheet width tends to zero. The Electric-Field Integral Equation, EFIE, leads then to a simplified problem where the rim currents are discarded, and the unknowns correspond to the sum of the currents over the top and bottom surfaces of the sheet [2]. The sheet appears modelled as an open surface - with zero thickness - that neglects the scattering contribution from the rims. Clearly, the thin-sheet approximation is advantageous in computational terms because of the drastic reduction of unknowns with respect to the full modelling of the structure as a closed surface. Also, the thin-sheet scheme has been used in many practical applications with little sacrifice of accuracy. However, in some cases, such as, the scattering analysis of PEC sheets with grazing incidences, the accuracy loss becomes especially evident. Recently, we have presented a new formulation for the efficient and accurate scattering analysis of plates with nonzero thickness under an arbitrary incidence [3]. In this paper, we extend such approach to the efficient scattering analysis of thick curved PEC sheets.

\section{THICK PLATE}

The scattering analysis of a free-standing PEC plate with nonzero thickness with the method-of-moment (MoM) discretization of the EFIE [4], leads to the following expression of the approximated scattered electric

$$
\begin{aligned}
\tilde{\boldsymbol{E}}^{s}(\boldsymbol{r})= & -j k \eta \sum_{n=1}^{N_{E}} c_{n} \iint_{Q_{n}} G\left(\boldsymbol{r}, \boldsymbol{r}^{\prime}\right) \boldsymbol{t}_{n}\left(\boldsymbol{r}^{\prime}\right) d S^{\prime} \\
& -j \frac{\eta}{k} \sum_{n=1}^{N_{E}} c_{n} \iint_{Q_{n}} \nabla G\left(\boldsymbol{r}, \boldsymbol{r}^{\prime}\right) \nabla^{\prime} \cdot \boldsymbol{t}_{n}\left(\boldsymbol{r}^{\prime}\right) d S^{\prime}
\end{aligned}
$$

where $N_{E}$ denotes the number edges arising from the discretization, the basis function $\boldsymbol{t}_{n}$ straddles $Q_{n}$, the pair of adjacent facets sharing the $n$-th edge, and $\left\{c_{n}\right\}$ represents the set of unknown coefficients. The parameters $k$ and $\eta$ denote, respectively, the wavenumber and the free-space impedance, whereas $G$ represents the free-space Green's function. Typically, low-order divergence-conforming sets of basis functions are adopted; namely, RWG [5] or rooftop [6], for triangular or rectangular meshes, respectively.

The EFIE relies on the application of the tangential electric-field boundary condition over the surface $S$ enclosing the plate

$$
\left.\tilde{\boldsymbol{E}}^{s}(\boldsymbol{r})\right|_{\tan , \boldsymbol{r} \in S}=-\left.\boldsymbol{E}^{i n c}(\boldsymbol{r})\right|_{\tan , \boldsymbol{r} \in S}
$$

where $\boldsymbol{E}^{\text {inc }}$ stands for the incident electric field. The resulting MoM-matrix system arises from testing (2) over a tessellation -triangular or rectangular- of $S$ with the adopted set of basis functions; that is,

$$
\begin{gathered}
\int_{Q_{m}} \boldsymbol{t}_{m}(r) \cdot \tilde{\boldsymbol{E}}^{s}(\boldsymbol{r}) d S=-\int_{Q_{m}} \boldsymbol{t}_{m}(r) \cdot \boldsymbol{E}^{i n c}(\boldsymbol{r}) d S \\
1 \leq m \leq N_{E}
\end{gathered}
$$

The scattering analysis of an object that is geometrically symmetric with respect to a plane of reference can be decomposed, with no accuracy loss, into two decoupled problems through the addition/subtraction, respectively, of pairs of field mirror contributions generated by the summation/subtraction of pairs of mirror source contributions [7]. The original MoM-problem in (3) is then converted into two mutually uncoupled matrix systems with roughly half the number of unknowns. Each of these systems captures the field or current contributions with either even or odd symmetry with respect to the reference plane. 
The analysis of a thick plate can be referenced for symmetry purposes to the mid-surface $S_{\mathrm{M}}$, which lies inside the plate at the same distance from the top and bottom faces (see Fig. 1). A symmetric mesh over the enclosing surface is required to group the current-expansion or field-testing basis functions in pairs of mirror functions. A subset of basis functions evolves along the borderline between halves, thereby with no symmetric peer. These single functions, as we call them, may show even or odd symmetry with respect to the plane of reference depending on the adopted scheme of meshing. The set of single basis functions need then to be accordingly aggregated as current-expansion or field-testing basis functions in the corresponding even- or odd-symmetric system. In this paper, we call the MoM-implementation derived from exploiting such symmetry properties in the standard MoM-approach in (3) as EFIE[sym].

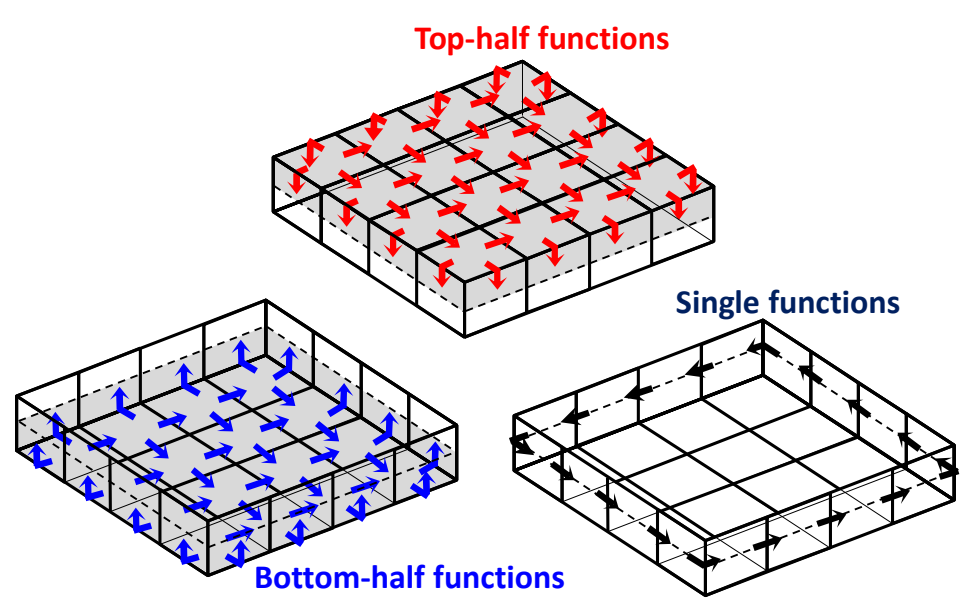

Fig. 1. Symmetric grouping of basis functions

Our thick-plate formulation [3] applies the standard tangential-electric condition of (2) over the $N_{R}$ pairs of facets sharing mesh edges that lie inside the plate rim or that match the outer boundary line of the rim (see Fig. 2). We then index $\left\{\boldsymbol{t}_{1}, \ldots, \boldsymbol{t}_{N_{R}}\right\}\left(\subset\left\{\boldsymbol{t}_{1}, \ldots, \boldsymbol{t}_{N_{E}}\right\}\right)$ as the subset of basis functions required for testing purposes over the rim mesh (see Fig. 2). On the other hand, unlike the standard MoM-scheme in (3), in our thick-plate approach no field conditions are imposed over the top and bottom plate faces. Instead, the following electric-field conditions are applied over the mid-surface $S_{\mathrm{M}}$

$$
\begin{gathered}
\left.\tilde{\boldsymbol{E}}^{s}(\boldsymbol{r})\right|_{\tan , \boldsymbol{r} \in S_{M}}=-\left.\boldsymbol{E}^{i n c}(\boldsymbol{r})\right|_{\tan , \boldsymbol{r} \in S_{M}} \\
\left.\frac{\partial}{\partial z}\left(\tilde{\boldsymbol{E}}^{s}(\boldsymbol{r})\right)\right|_{\tan , \boldsymbol{r} \in S_{M}}=-\left.\frac{\partial}{\partial z}\left(\boldsymbol{E}^{i n c}(\boldsymbol{r})\right)\right|_{\tan , \boldsymbol{r} \in S_{M}}
\end{gathered}
$$

and $S_{\mathrm{M}}$ lies on the xy plane, inside the plate. We test the field conditions in (4) and (5) with the set of basis functions $\left\{\boldsymbol{t}_{1}^{M} \ldots \boldsymbol{t}_{N_{M}}^{M}\right\}$ associated with the $N_{M}$ interior edges of the mesh at $S_{\mathrm{M}}$, a translated copy of the meshes over the top- and bottom-faces (see Fig. 2). Overall, the resulting MoMsystem yields

$$
\begin{aligned}
& \iint_{Q_{r}} \boldsymbol{t}_{r} \cdot \tilde{\boldsymbol{E}}^{s}(\boldsymbol{r}) d S=-\iint_{Q_{r}} \boldsymbol{t}_{r} \cdot \boldsymbol{E}^{i n c}(\boldsymbol{r}) d S \\
& \iint_{Q_{m}^{M}} \boldsymbol{t}_{m}^{M} \cdot \tilde{\boldsymbol{E}}^{s}(\boldsymbol{r}) d S=-\iint_{Q_{m}^{M}} \boldsymbol{t}_{m}^{M} \cdot \boldsymbol{E}^{i n c}(\boldsymbol{r}) d S \\
& \iint_{Q_{m}^{M}} \boldsymbol{t}_{m}^{M} \cdot \frac{\partial}{\partial z}\left(\tilde{\boldsymbol{E}}^{s}(\boldsymbol{r})\right) d S=-\iint_{Q_{m}^{M}} \boldsymbol{t}_{m}^{M} \cdot \frac{\partial}{\partial z}\left(\boldsymbol{E}^{i n c}(\boldsymbol{r})\right) d S \\
& 1 \leq r \leq N_{R}, \quad 1 \leq m \leq N_{M}
\end{aligned}
$$

where $Q_{m}^{M}$ represents the duplet of facets (rectangular or triangular) that share the $m$-th edge inside the mesh at $S_{\mathrm{M}}$.

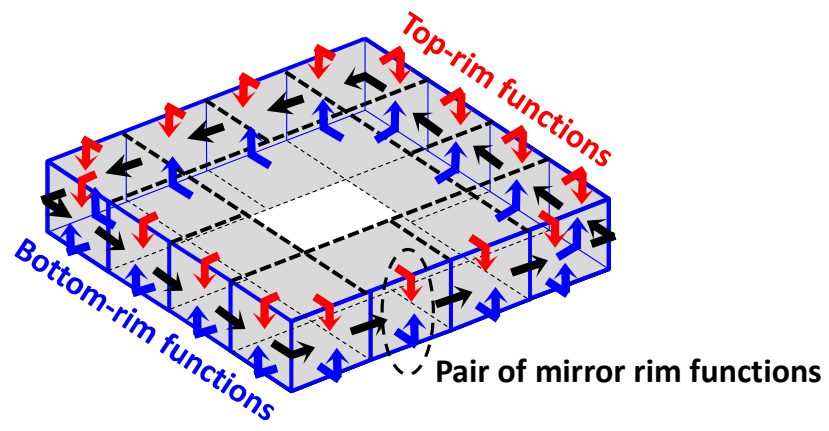

(a)

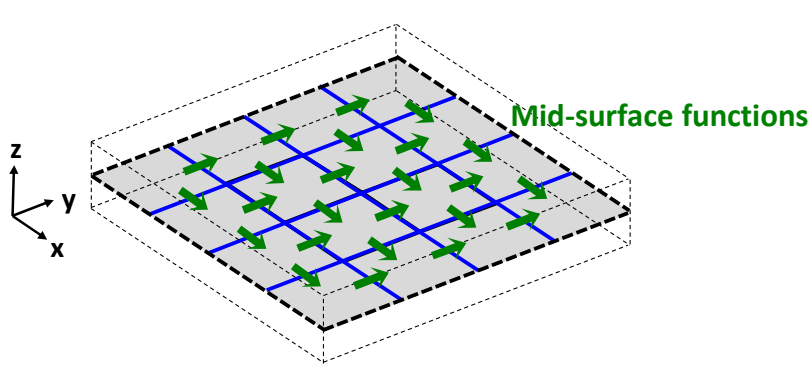

(b)

Fig. 2. Thick-plate implementation for a plate with rectangular grid: (a) Rim Testing; (b) Mid-surface testing.

In the expansion of the current, hence, each basis function in the top half of the plate has a mirror function at the bottom half (see Fig. 1). We then rearrange these symmetric basis functions into two sets, which arise from either summing or subtracting the symmetric pairs of basis functions. These sum and difference source sets appear exclusively coupled with the field conditions (7) and (8), respectively. Also, they appear only associated with the outcome of adding or subtracting, respectively, the field condition (6) over symmetric pairs of top- and bottom rim testing functions (see Fig. 2). Our thick-plate scheme, EFIE[thick], leads to two mutually uncoupled matrix systems, in an analogous manner as EFIE[sym]. Both EFIE[sym] and EFIE[thick] schemes turn out more efficient 
than the standard MoM-solution of (3), especially for problems with a large amount of unknowns, and are easily amenable to parallelization. However, the process of matrix generation and manipulation appears more elaborate and awkward in the scheme EFIE[sym] than in EFIE[thick]. Indeed, EFIE[sym] assumes the search and reordering of the basis functions in pairs of mirror basis functions over the top-face and bottom-face meshes. This becomes computationally demanding for plates with electrically large faces, especially, as it often happens, if the mesh is generated by another party. In contrast, EFIE[thick] circumvents the management of two meshes, over the top and bottom faces, through the adoption of one single mesh, over the mid-surface (see Fig. 2), where the top-face and bottom-face mirror source contributions can be automatically assigned and where the field tests (7) or (8) are applied.

\section{THICK CURVED SHEET}

Consider the scattering analysis of a free-standing curved sheet with nonzero thickness under an incident plane wave. We model the curved sheets such that (i) the rims of the thick sheet impinge perpendicularly on the top and bottom surfaces of the sheet, and (ii) the top and bottom surfaces are parallel (see Fig. 3). Just like in the thick-plate approach, the mid-surface of the curved sheet lies inside the structure and is equidistant to the top and bottom surfaces (see Fig. 3-(b)).

In the analysis of thick curved sheets, we explore the application of the schemes EFIE[sym] and EFIE[thick], successfully implemented for the scattering analysis of thick plates [3]. Pairs of mirror source or testing basis functions can be also defined in the thick curved-sheet analysis, in an analogous manner as done in the thick-plate schemes. However, now, as shown clearly in Fig. 3, unlike the thick plate, the mid-surface and curved-sheet top or bottom surfaces are not translated copies (their radii of curvature are slightly different). In consequence, the crucial symmetry properties that justify the decomposition of the original MoM-problem into two decoupled systems, with reduced number of unknowns, strictly speaking do not appear valid anymore. Some loss of accuracy is then expected for the thick curved sheet analysis with the EFIE[sym] or EFIE[thick] schemes. As a matter of fact, the thick plate contains surfaces with infinite radius of curvature. Therefore, intuitively speaking, it appears reasonable that the observed inaccuracy in the thick curved sheet analysis decreases as the radii of curvature of the involved surfaces grow or as the thickness of the curved sheet diminishes. In both cases, the top and bottom surfaces differ less, and therefore approach better the top-bottom symmetry paradigm.

The thick curved-sheet approach follows the guidelines described in (6)-(8) for the thick plate, which results in the following matrix system

$$
\iint_{Q_{r}} \boldsymbol{t}_{r} \cdot \tilde{\boldsymbol{E}}^{s}(\boldsymbol{r}) d S=-\iint_{Q_{r}} \boldsymbol{t}_{r} \cdot \boldsymbol{E}^{i n c}(\boldsymbol{r}) d S
$$

$$
\begin{gathered}
\iint_{Q_{m}^{M}} \boldsymbol{t}_{m}^{M} \cdot \tilde{\boldsymbol{E}}^{s}(\boldsymbol{r}) d S=-\iint_{Q_{m}^{M}} \boldsymbol{t}_{m}^{M} \cdot \boldsymbol{E}^{i n c}(\boldsymbol{r}) d S \\
\iint_{Q_{m}^{M}} \boldsymbol{t}_{m}^{M} \cdot \frac{\partial}{\partial n}\left(\tilde{\boldsymbol{E}}^{s}(\boldsymbol{r})\right) d S=-\iint_{Q_{m}^{M}} \boldsymbol{t}_{m}^{M} \cdot \frac{\partial}{\partial n}\left(\boldsymbol{E}^{i n c}(\boldsymbol{r})\right) d S \\
1 \leq r \leq N_{R}, \quad 1 \leq m \leq N_{M}
\end{gathered}
$$

where $n$ denotes the normal coordinate, defined locally over each facet belonging to the $m$-th testing facet-duplet $Q_{m}^{M}$ (see Fig. 3-(b)).

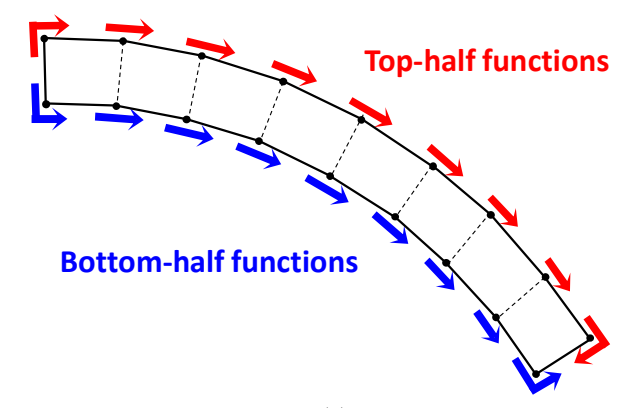

(a)

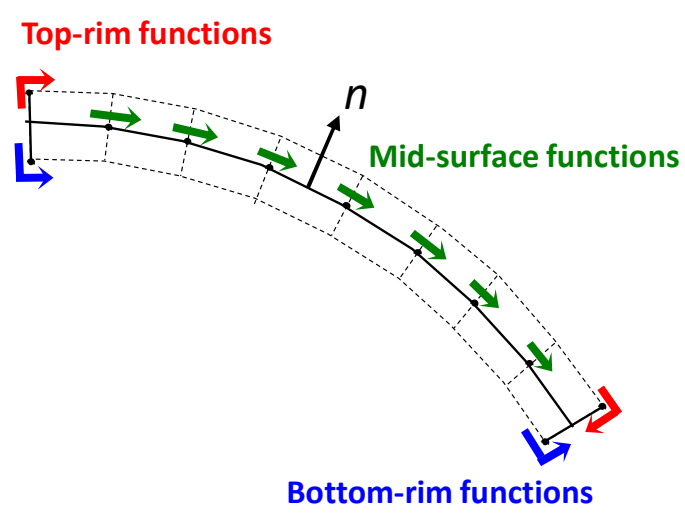

(b)

Fig. 3. Thick curved sheet discretization: (a) Symmetric grouping; (b) Rim and Mid-surface testing

\section{RESULTS}

In Figs. 5 and 6, we show RCS results computed with the schemes EFIE[sym] and EFIE[thick] for several curved sheets with nonzero thickness. For comparison purposes, we display also MoM-results obtained from the full modelling of the object, as a closed surface, EFIE[full], or through the thin-sheet approximation, EFIE[thin], which assumes zero thickness. The types of thick curved sheets adopted in Figs. 5 and 6 are spherical caps with outer radii of $2 \mathrm{~m}$ or $4 \mathrm{~m}$ and aperture angles of $40^{\circ}$ and $30^{\circ}$, respectively (see Fig. 4). The thickness of both spherical caps is $0.02 \mathrm{~m}$. The impinging plane wave is $\mathrm{x}$-propagating and z-polarized in all cases. The working wavelength is $1 \mathrm{~m}$. 


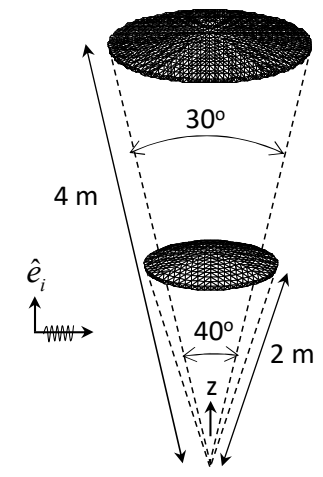

Fig. 4. Spherical caps with radii of curvature and aperture angles, respectively, of $4 \lambda$ and $30^{\circ}$ (top) and $2 \lambda$ and $40^{\circ}$ (bottom). The triangular meshes adopted contain 4290 facets (top) or 1386 facets (bottom). The thickness is $0.02 \mathrm{~m}$ in both cases.

In view of Figs. 5 and 6, the implementations EFIE[sym] and EFIE[thick] show good match with the full MoMsolution. Under the adopted grazing incidence (see Fig. 4), the thin-surface approximation EFIE[thin] shows evident inaccuracies.

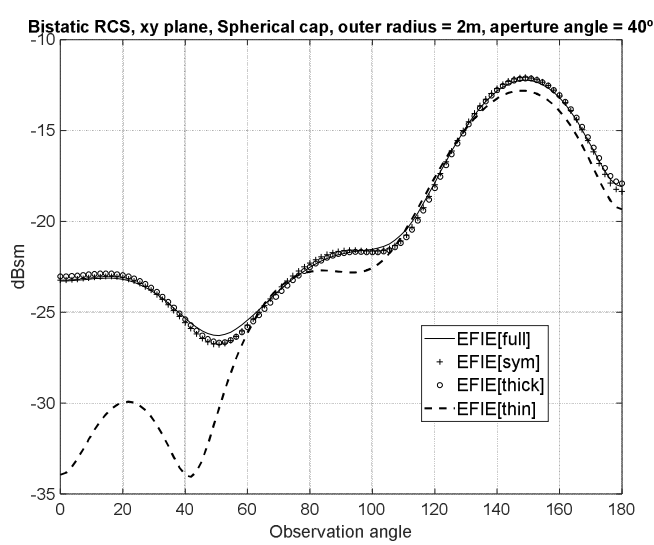

Fig. 5. RCS cut over the H-plane computed for several EFIEimplementations and an impinging z-polarized plane wave for a spherical cap with outer radius of $2 \lambda$, aperture angle of $40^{\circ}$ and thickness of $0.02 \mathrm{~m}$.

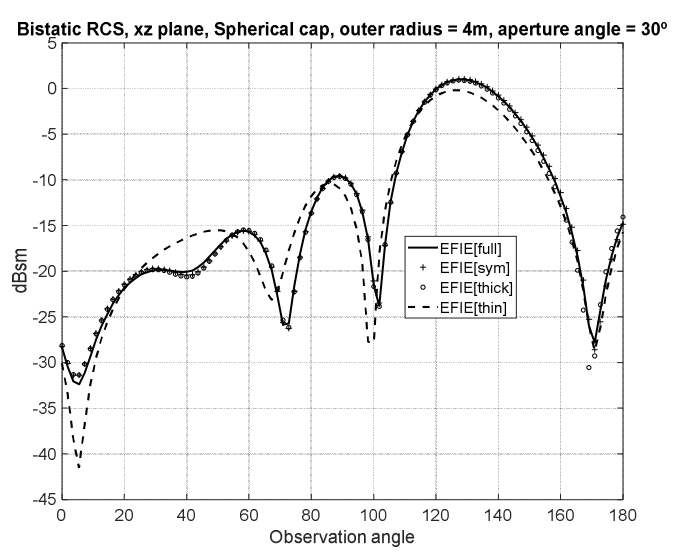

Fig. 6. RCS cut over the E-plane computed for several EFIEimplementations and an impinging z-polarized plane wave for a spherical cap with outer radius of $4 \lambda$, aperture angle of $30^{\circ}$ and thickness of $0.02 \mathrm{~m}$.
The computational load in the full MoM-solution involves 2079 and 6435 unknowns, respectively, for the tested spherical caps with radii $2 \lambda$ and $4 \lambda$. In contrast, the thin-surface approach reduces the problem down to 819 and 2717 unknowns, respectively. The schemes EFIE[thick] and EFIE[sym] offer good accuracy after solving two concurrent systems of 1071 and 1008 unknowns for the $2 \lambda$-case or of 3289 and 3146 unknowns for the $4 \lambda$-case.

\section{CONCLUSIONS}

Our thick-surface implementation shows competitive accuracy with respect to the full MoM EFIE-solution for the tested thick slightly curved sheets (spherical caps) under grazing incidence angles, where the thin-surface approach fails. The thick-surface solutions require the computation of two systems with half the number of unknowns of the original MoM-problem. Our thick-surface implementation simplifies the matrix generation and manipulation through the testing over a reference surface inside the curved sheet. As expected, our tests confirm that good accuracy is obtained for sheet thicknesses rather smaller than observed in the accurate scattering analysis of thick plates [3].

\section{ACKNOWLEDGMENT}

This work was supported by FEDER and the "Spanish Plan Estatal de Investigación Científica y Técnica y de Innovación", under projects: TEC2017-84817-C2-2-R/ AEI/ 10.13039/501100011033, TEC2016-78028-C3-1-P/AEI/10.1 3039/501100011033, PID2019-107885GB-C31/AEI/10.130 $39 / 501100011033$ and the Unidad de Excelencia Maria de Maeztu MDM-2016-0600/AEI/10.13039/501100011033, which is financed by the Agencia Estatal de Investigación, Spain, and Catalan Research Group 2017 SGR 219.

\section{REFERENCES}

[1] Branko M. Kolundzija and Antonije R. Djordjevic, Electromagnetic Modeling of Composite Metallic and Dielectric Structures, Boston: Artech House, 2002.

[2] Edward H. Newman and Mark R. Schrote, "On the Current Distribution for Open Surfaces," IEEE Trans. Antennas Propag., vol. AP-31, no. 3, pp. 515-518, May 1983

[3] Eduard Ubeda, Ivan Sekulic, and Juan M. Rius, "Efficient and accurate electromagnetic scattering analysis of perfectly conducting thick plates," presented at the IEEE Int. Symp. Antennas and Propagation, Atlanta, GA, USA, Jul. 7-12, 2019.

[4] Roger F. Harrington, Field Computation by Moment Method, New York, NY, USA: Macmillan, 1968.

[5] Sadasiva M. Rao, Donald R. Wilton, and Allen W. Glisson, "Electromagnetic scattering by surfaces of arbitrary shape," IEEE Trans. Antennas Propag., vol. 30, no. 3, pp. 409-418, May 1982.

[6] Allen W. Glisson, and Donald R. Wilton, "Simple and Efficient Numerical Methods for Problems of Electromagnetic Radiation and Scattering from Surfaces", IEEE Trans. Antennas Propag., vol. AP28, no. 5, pp. 593-603, Sept. 1980

[7] Roy McWeeny, Symmetry: An Introduction to Group Theory and Its Applications, Mineola, New York: Dover Publications, inc., 2002. 